\title{
Interference of Neutral Kaons in the Hadronic Decays of the $\mathrm{Z}^{0}$
}

\author{
DELPHI Collaboration
}

\begin{abstract}
$\mathrm{K}_{\mathrm{S}}^{0} \mathrm{~K}_{\mathrm{S}}^{0}$ correlations have been studied in a sample of 717,511 hadronic events collected by the DELPHI detector at LEP during 1992. An enhancement is found in the production of pairs of $K_{S}^{0}$ of similar momenta, as compared with a Monte Carlo simulated reference sample. The measured values for the strength of the correlation and the radius of the emitting source of kaons are $\lambda=1.13 \pm 0.54($ stat $) \pm 0.23$ (syst) and $r=0.90 \pm 0.19($ stat $) \pm 0.10($ syst $) \mathrm{fm}$. This enhancement is consistent with the hypothesis that $K_{S}^{0} K_{S}^{0}$ pairs display an enhancement, regardless of whether they come from a $\mathrm{K}^{0} \overline{\mathrm{K}}^{0}$ or from a $\mathrm{K}^{0} \mathrm{~K}^{0}$ $\left(\overline{\mathrm{K}}^{0} \overline{\mathrm{K}}^{0}\right)$ system.
\end{abstract}




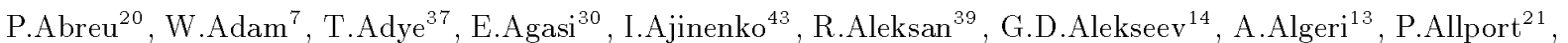
S.Almehed $^{23}$, S.J.Alvsvaag ${ }^{4}$, U.Amaldi ${ }^{7}$, A.Andreazza ${ }^{27}$, P.Antilogus ${ }^{24}$, W-D.Apel ${ }^{15}$, R.J.Apsimon ${ }^{37}$, Y.Arnoud $^{39}$, B.Asman ${ }^{45}$, J-E.Augustin ${ }^{18}$, A.Augustinus ${ }^{30}$, P.Baillon ${ }^{7}$, P.Bambade ${ }^{18}$, F.Barao ${ }^{20}$, R.Barate ${ }^{12}$,

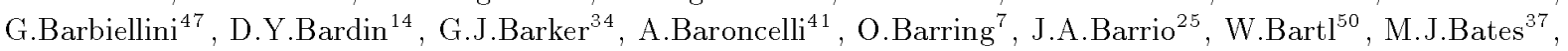
M.Battaglia $^{13}$, M.Baubillier ${ }^{22}$, M.Begalli ${ }^{36}$, P.Beilliere ${ }^{6}, \quad$ P.Beltran $^{9}$, A.C.Benvenuti ${ }^{5}, \quad$ M.Berggren $^{18}$, D.Bertrand $^{2}$, F.Bianchi ${ }^{46}$, M.Bigi ${ }^{46}$, M.S.Bilenky ${ }^{14}$, P.Billoir ${ }^{22}$, J.Bjarne ${ }^{23}$, D.Bloch ${ }^{8}$, J.Blocki ${ }^{51}$, M.Blume ${ }^{52}$, S.Blyth $^{34}$, V.Bocci ${ }^{38}$, P.N.Bogolubov ${ }^{14}$, T.Bolognese ${ }^{39}$, M.Bonesini ${ }^{27}$, W.Bonivento ${ }^{27}$, P.S.L.Booth ${ }^{21}$, G.Borisov $^{43}$, H.Borner ${ }^{7}$, C.Bosio ${ }^{41}$, B.Bostjancic ${ }^{44}$, S.Bosworth $^{34}$, O.Botner ${ }^{48}$, B.Bouquet ${ }^{18}$, C.Bourdarios ${ }^{18}$, T.J.V.Bowcock ${ }^{21}$, M.Bozzo ${ }^{11}$, S.Braibant ${ }^{2}$, P.Branchini ${ }^{41}$, K.D.Brand ${ }^{35}$, T.Brenke ${ }^{52}$, R.A.Brenner ${ }^{7}$, H.Briand ${ }^{22}$, C.Bricman $^{2}$, L.Brillault ${ }^{22}$, R.C.A.Brown ${ }^{7}$, P.Bruckman ${ }^{16}$, J-M.Brunet ${ }^{6}$, A.Budziak ${ }^{16}$, L.Bugge ${ }^{32}$, T.Buran ${ }^{32}$, H.Burmeister ${ }^{7}$, A.Buys ${ }^{7}$, J.A.M.A.Buytaert ${ }^{7}$, M.Caccia ${ }^{27}$, M.Calvi ${ }^{27}$, A.J.Camacho Rozas ${ }^{42}$, R.Campion ${ }^{21}$,

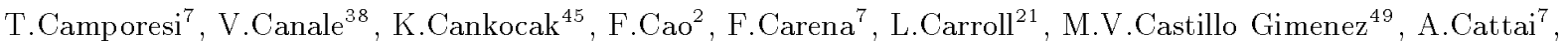
F.R.Cavallo $^{5}$ L.Cerrito $^{38}$, V.Chabaud ${ }^{7}$, A.Chan ${ }^{1}$, M.Chapkin ${ }^{43}$, Ph.Charpentier ${ }^{7}$, J.Chauveau ${ }^{22}$, P.Checchia ${ }^{35}$, G.A.Chelkov ${ }^{14}$, L.Chevalier ${ }^{39}$, P.Chliapnikov ${ }^{43}$, V.Chorowicz ${ }^{22}$, J.T.M.Chrin ${ }^{49}$, V.Cindro ${ }^{44}$, P.Collins ${ }^{34}$, J.L.Contreras ${ }^{18}$, R.Contri ${ }^{11}$, E.Cortina ${ }^{49}$, G.Cosme $^{18}$, F.Couchot ${ }^{18}$, H.B.Crawley ${ }^{1}$, D.Crennell ${ }^{37}$, G.Crosetti $^{11}$, J.Cuevas Maestro ${ }^{33}$, S.Czellar ${ }^{13}$, E.Dahl-Jensen ${ }^{28}$, J.Dahm ${ }^{52}$, B.Dalmagne ${ }^{18}$, M.Dam ${ }^{32}$, G.Damgaard ${ }^{28}$, E.Daubie $^{2}$, A.Daum ${ }^{15}$, P.D.Dauncey ${ }^{7}$, M.Davenport ${ }^{7}$, J.Davies ${ }^{21}$, W.Da Silva ${ }^{22}$, C.Defoix ${ }^{6}$, P.Delpierre ${ }^{26}$, N.Demaria ${ }^{46}$ A.De Angelis ${ }^{7}$, H.De Boeck ${ }^{2}$, W.De Boer ${ }^{15}$, S.De Brabandere ${ }^{2}$, C.De Clercq ${ }^{2}$, M.D.M.De Fez Laso ${ }^{49}$, C.De La Vaissiere ${ }^{22}$, B.De Lotto ${ }^{47}$, A.De Min ${ }^{27}$, H.Dijkstra ${ }^{7}$, L.Di Ciaccio ${ }^{38}$, F.Djama ${ }^{8}$, J.Dolbeau $^{6}$, M.Donszelmann ${ }^{7}$, K.Doroba ${ }^{51}$, M.Dracos $^{8}$, J.Drees ${ }^{52}$, M.Dris ${ }^{31}$, Y.Dufour ${ }^{7}$, F.Dupont ${ }^{12}$, D.Edsall ${ }^{1}$, L-O.Eek $^{48}$, P.A.-M.Eerola ${ }^{7}$, R.Ehret ${ }^{15}$, T.Ekelof ${ }^{48}$, G.Ekspong ${ }^{45}$, A.Elliot Peisert ${ }^{7}$, M.Elsing ${ }^{52}$, J-P.Engel ${ }^{8}$, N.Ershaidat ${ }^{22}$, M.Espirito Santo ${ }^{20}$, V.Falaleev ${ }^{43}$, D.Fassouliotis ${ }^{31}$, M.Feindt ${ }^{7}$, A.Fenyuk ${ }^{43}$, A.Ferrer ${ }^{49}$, T.A.Filippas ${ }^{31}$, A.Firestone ${ }^{1}$, H.Foeth ${ }^{7}$, E.Fokitis ${ }^{31}$, F.Fontanelli ${ }^{11}$, K.A.J.Forbes ${ }^{21}$, J-L.Fousset ${ }^{26}$, S.Francon ${ }^{24}$, B.Franek $^{37}$, P.Frenkiel ${ }^{6}$, D.C.Fries ${ }^{15}$, A.G.Frodesen ${ }^{4}$, R.Fruhwirth ${ }^{50}$, F.Fulda-Quenzer ${ }^{18}$, H.Furstenau ${ }^{15}$, J.Fuster ${ }^{7}$, D.Gamba ${ }^{46}$, M.Gandelman ${ }^{17}$, C.Garcia $^{49}$, J.Garcia $^{42}$, C.Gaspar $^{7}$, U.Gasparini ${ }^{35}$, Ph.Gavillet ${ }^{7}$, E.N.Gazis ${ }^{31}$, J-P.Gerber ${ }^{8}$, P.Giacomelli ${ }^{7}$ ， D.Gillespie ${ }^{7}$, R.Gokieli ${ }^{51}$ ， B.Golob ${ }^{44}$, V.M.Golovatyuk ${ }^{14}$, J.J.Gomez Y Cadenas ${ }^{7}$, G.Gopal ${ }^{37}$, L.Gorn ${ }^{1}$, M.Gorski ${ }^{51}$, V.Gracco ${ }^{11}$, A.Grant ${ }^{7}$, F.Grard ${ }^{2}$, E.Graziani ${ }^{41}$, G.Grosdidier $^{18}$, E.Gross ${ }^{7}$, B.Grossetete ${ }^{22}$, P.Gunnarsson ${ }^{45}$, J.Guy ${ }^{37}$, U.Haedinger ${ }^{15}$, F.Hahn ${ }^{52}$, M.Hahn ${ }^{45}$, S.Hahn $^{52}$, S.Haider ${ }^{30}$, Z.Hajduk ${ }^{16}$, A.Hakansson ${ }^{23}$, A.Hallgren ${ }^{48}$, K.Hamacher ${ }^{52}$, G.Hamel De Monchenault ${ }^{39}$, W.Hao $^{30}$, F.J.Harris ${ }^{34}$, V.Hedberg ${ }^{23}$, T.Henkes ${ }^{7}$, R.Henriques ${ }^{20}$, J.J.Hernandez ${ }^{49}$, J.A.Hernando ${ }^{49}$, P.Herquet $^{2}$, H.Herr $^{7}$, T.L.Hessing ${ }^{21}$, I.Hietanen ${ }^{13}$, C.O.Higgins ${ }^{21}$, E.Higon ${ }^{49}$, H.J.Hilke ${ }^{7}$, T.S.Hill ${ }^{1}$, S.D.Hodgson ${ }^{34}$, T.Hofmokl $^{51}$, S-O.Holmgren ${ }^{45}$, P.J.Holt ${ }^{34}$, D.Holthuizen ${ }^{30}$, P.F.Honore ${ }^{6}$, M.Houlden ${ }^{21}$, J.Hrubec ${ }^{50}$, K.Huet $^{2}$, K.Hultqvist $^{45}$, P.Ioannou ${ }^{3}$, P-S.Iversen ${ }^{4}$, J.N.Jackson ${ }^{21}$, R.Jacobsson ${ }^{45}$, P.Jalocha ${ }^{16}$, G.Jarlskog ${ }^{23}$, P.Jarry ${ }^{39}$, B.Jean-Marie ${ }^{18}$, E.K.Johansson ${ }^{45}$, M.Jonker ${ }^{7}$, L.Jonsson ${ }^{23}$, P.Juillot ${ }^{8}$, G.Kalkanis ${ }^{3}$, G.Kalmus ${ }^{37}$, F.Kapusta ${ }^{22}$, M.Karlsson ${ }^{45}$, E.Karvelas ${ }^{9}$, S.Katsanevas ${ }^{3}$, E.C.Katsoufis ${ }^{31}$, R.Keranen ${ }^{7}$, B.A.Khomenko ${ }^{14}$, N.N.Khovanski ${ }^{14}$, B.King $^{21}$, N.J.Kjaer ${ }^{7}$, H.Klein ${ }^{7}$, A.Klovning ${ }^{4}$, P.Kluit ${ }^{30}$, A.Koch-Mehrin ${ }^{52}$, J.H.Koehne ${ }^{15}$, B.Koene ${ }^{30}$, P.Kokkinias $^{9}$, M.Koratzinos ${ }^{32}$, A.V.Korytov ${ }^{14}$, V.Kostioukhine ${ }^{43}$, C.Kourkoumelis ${ }^{3}$, O.Kouznetsov ${ }^{14}$, P.H.Kramer ${ }^{52}$, M.Krammer ${ }^{50}$, C.Kreuter ${ }^{15}$, J.Krolikowski ${ }^{51}$, I.Kronkvist ${ }^{23}$, W.Kucewicz ${ }^{16}$, K.Kulka $^{48}$, K.Kurvinen $^{13}$, C.Lacasta ${ }^{49}$, C.Lambropoulos ${ }^{9}$, J.W.Lamsa ${ }^{1}$, L.Lanceri ${ }^{47}$, P.Langefeld ${ }^{52}$, V.Lapin ${ }^{43}$, I.Last $^{21}$, J-P.Laugier $^{39}$, R.Lauhakangas ${ }^{13}$, G.Leder ${ }^{50}$, F.Ledroit ${ }^{12}$, R.Leitner ${ }^{29}$, Y.Lemoigne ${ }^{39}$, J.Lemonne ${ }^{2}$, G.Lenzen $^{52}$, V.Lepeltier $^{18}$, J.M.Levy ${ }^{8}$, E.Lieb $^{52}$, D.Liko ${ }^{50}$, J.Lindgren $^{13}, \quad$ R.Lindner $^{52}, \quad$ A.Lipniacka $^{18}, \quad$ I.Lippi $^{35}$, B.Loerstad $^{23}$, M.Lokajicek ${ }^{10}$, J.G.Loken ${ }^{34}$, A.Lopez-Fernandez ${ }^{7}$, M.A.Lopez Aguera ${ }^{42}$, M.Los ${ }^{30}$, D.Loukas ${ }^{9}$,

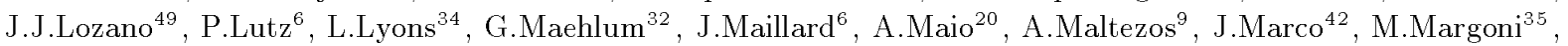
J-C.Marin ${ }^{7}$, C.Mariotti ${ }^{41}$, A.Markou ${ }^{9}$, T.Maron ${ }^{52}$, S.Marti ${ }^{49}$, C.Martinez-Rivero ${ }^{42}$, F.Martinez-Vidal ${ }^{49}$, F.Matorras $^{42}$, C.Matteuzzi ${ }^{27}$, G.Matthiae ${ }^{38}$, M.Mazzucato $^{35}$, M.Mc Cubbin ${ }^{21}$, R.Mc Kay ${ }^{1}$, R.Mc Nulty ${ }^{21}$, J.Medbo $^{48}$, C.Meroni ${ }^{27}$, W.T.Meyer ${ }^{1}$, M.Michelotto $^{35}$, I.Mikulec ${ }^{50}$, L.Mirabito ${ }^{24}$, W.A.Mitaroff ${ }^{50}$, G.V.Mitselmakher ${ }^{14}$, U.Mjoernmark ${ }^{23}$, T.Moa ${ }^{45}$, R.Moeller ${ }^{28}$, K.Moenig ${ }^{7}$, M.R.Monge ${ }^{11}$, P.Morettini ${ }^{11}$, H.Mueller $^{15}$, W.J.Murray ${ }^{37}$, B.Muryn ${ }^{16}$, G.Myatt ${ }^{34}$, F.Naraghi ${ }^{12}$, F.L.Navarria ${ }^{5}$, P.Negri ${ }^{27}$, S.Nemecek ${ }^{10}$, W.Neumann ${ }^{52}$, N.Neumeister ${ }^{50}$, R.Nicolaidou ${ }^{3}$, B.S.Nielsen ${ }^{28}$, P.E.S.Nilsen ${ }^{4}$, P.Niss ${ }^{45}$, A.Nomerotski ${ }^{35}$, V.Obraztsov $^{43}$, A.G.Olshevski ${ }^{14}$, R.Orava ${ }^{13}$, A.Ostankov ${ }^{43}$, K.Osterberg ${ }^{13}$, A.Ouraou ${ }^{39}$, P.Paganini ${ }^{18}$, M.Paganoni $^{27}$, R.Pain ${ }^{22}$, H.Palka ${ }^{16}$, Th.D.Papadopoulou ${ }^{31}$, L.Pape ${ }^{7}$, F.Parodi ${ }^{11}$, A.Passeri ${ }^{41}$, M.Pegoraro ${ }^{35}$, J.Pennanen ${ }^{13}$, L.Peralta ${ }^{20}$, H.Pernegger ${ }^{50}$, M.Pernicka ${ }^{50}$, A.Perrotta ${ }^{5}$, C.Petridou ${ }^{47}$, A.Petrolini ${ }^{11}$, G.Piana ${ }^{11}$, F.Pierre $^{39}$, M.Pimenta ${ }^{20}$, S.Plaszczynski ${ }^{18}$, O.Podobrin ${ }^{15}$, M.E.Pol ${ }^{17}$, G.Polok ${ }^{16}$, P.Poropat ${ }^{47}$, V.Pozdniakov ${ }^{14}$, M.Prest ${ }^{47}$, P.Privitera ${ }^{38}$, A.Pullia ${ }^{27}$, D.Radojicic ${ }^{34}$, S.Ragazzi ${ }^{27}$, H.Rahmani ${ }^{31}$, J.Rames ${ }^{10}$, P.N.Ratoff ${ }^{19}$, A.L.Read ${ }^{32}$, M.Reale ${ }^{52}$, P.Rebecchi ${ }^{18}$, N.G.Redaelli ${ }^{27}$, M.Regler ${ }^{50}$, D.Reid ${ }^{7}$, P.B.Renton ${ }^{34}$, L.K.Resvanis ${ }^{3}$, F.Richard ${ }^{18}$, J.Richardson ${ }^{21}$, J.Ridky ${ }^{10}$, G.Rinaudo ${ }^{46}$, A.Romero $^{46}$, I.Roncagliolo ${ }^{11}$, P.Ronchese ${ }^{35}$, C.Ronnqvist $^{13}$, E.I.Rosenberg ${ }^{1}$, E.Rosso ${ }^{7}$, P.Roudeau ${ }^{18}$, T.Rovelli ${ }^{5}$, W.Ruckstuhl ${ }^{30}$, V.Ruhlmann-Kleider ${ }^{39}$, A.Ruiz $^{42}$, K.Rybicki ${ }^{16}$, A.Rybin ${ }^{43}$, H.Saarikko ${ }^{13}$, Y.Sacquin ${ }^{39}$, G.Sajot ${ }^{12}$, J.Salt ${ }^{49}$, J.Sanchez ${ }^{25}$, M.Sannino ${ }^{1140}$, 
S.Schael ${ }^{7}$, H.Schneider ${ }^{15}$, M.A.E.Schyns ${ }^{52}$, G.Sciolla ${ }^{46}$, F.Scuri $^{47}$, A.M.Segar ${ }^{34}$, A.Seitz ${ }^{15}$, R.Sekulin $^{37}$, M.Sessa ${ }^{47}$, R.Seufert ${ }^{15}$, R.C.Shellard ${ }^{36}$, I.Siccama ${ }^{30}$, P.Siegrist ${ }^{39}$, S.Simonetti ${ }^{11}$, F.Simonetto ${ }^{35}$, A.N.Sisakian ${ }^{14}$, G.Skjevling ${ }^{32}$, G.Smadja ${ }^{39,24}$, N.Smirnov ${ }^{43}$, O.Smirnova ${ }^{14}$, G.R.Smith ${ }^{37}$, R.Sosnowski ${ }^{51}$, D.Souza-Santos ${ }^{36}$, T.Spassov $^{20}$, E.Spiriti ${ }^{41}$, S.Squarcia ${ }^{11}$, C.Stanescu ${ }^{41}$, S.Stapnes ${ }^{32}$, G.Stavropoulos ${ }^{9}$, F.Stichelbaut ${ }^{2}$, A.Stocchi $^{18}$, J.Strauss $^{50}$, J.Straver ${ }^{7}$, R.Strub ${ }^{8}$, B.Stugu ${ }^{4}$, M.Szczekowski ${ }^{7}$, M.Szeptycka ${ }^{51}$, P.Szymanski ${ }^{51}$, T.Tabarelli ${ }^{27}$, O.Tchikilev ${ }^{43}$, G.E.Theodosiou ${ }^{9}$. A.Tilquin ${ }^{26}$. J.Timmermans ${ }^{30}$, V.G.Timofeev ${ }^{14}$, L.G.Tkatchev ${ }^{14}$, T.Todorov ${ }^{8}$, D.Z.Toet ${ }^{30}$, O.Toker ${ }^{13}$, A.Tomaradze ${ }^{2}$, B.Tome ${ }^{20}$, E.Torassa ${ }^{46}$, L.Tortora ${ }^{41}$, D.Treille ${ }^{7}$, W.Trischuk ${ }^{7}$, G.Tristram ${ }^{6}$, C.Troncon ${ }^{27}$, A.Tsirou ${ }^{7}$, E.N.Tsyganov ${ }^{14}$, M.Turala $^{16}$, M-L.Turluer ${ }^{39}$, T.Tuuva ${ }^{13}$, I.A.Tyapkin ${ }^{22}$, M.Tyndel $^{37}$, S.Tzamarias ${ }^{21}$, B.Ueberschaer ${ }^{52}$, S.Ueberschaer ${ }^{52}$, O.Ullaland ${ }^{7}$, G.Valenti ${ }^{5}$, E.Vallazza ${ }^{7}$, J.A.Valls Ferrer ${ }^{49}$ C.Vander Velde ${ }^{2}$ G.W.Van Apeldoorn ${ }^{30}$, P.Van Dam ${ }^{30}$, M.Van Der Heijden ${ }^{30}$, W.K.Van Doninck ${ }^{2}$, J.Van Eldik ${ }^{30}$, P.Vaz ${ }^{7}$, G.Vegni ${ }^{27}$, L.Ventura ${ }^{35}$, W.Venus ${ }^{37}$, F.Verbeure ${ }^{2}$, M.Verlato $^{35}$, L.S.Vertogradov ${ }^{14}$, D.Vilanova ${ }^{39}$, P.Vincent ${ }^{24}$, L.Vitale ${ }^{47}$, E.Vlasov ${ }^{43}$, A.S.Vodopyanov ${ }^{14}$, M.Vollmer ${ }^{52}$, M.Voutilainen $^{13}$, V.Vrba ${ }^{41}$, H.Wahlen ${ }^{52}$, C.Walck ${ }^{45}$, F.Waldner ${ }^{47}$, A.Wehr ${ }^{52}$, M.Weierstall ${ }^{52}$, P.Weilhammer ${ }^{7}$, A.M.Wetherell ${ }^{7}$, J.H.Wickens ${ }^{2}$, M.Wielers ${ }^{15}$, G.R.Wilkinson ${ }^{34}$, W.S.C.Williams ${ }^{34}$, M.Winter ${ }^{8}$, G.Wormser ${ }^{18}$, K.Woschnagg $^{48}$, A.Zaitsev ${ }^{43}$, A.Zalewska ${ }^{16}$, D.Zavrtanik ${ }^{44}$, E.Zevgolatakos ${ }^{9}$, N.I.Zimin ${ }^{14}$, M.Zito $^{39}$, D.Zontar $^{44}$, R.Zuberi $^{34}$, G.Zumerle ${ }^{35}$, J.Zuniga ${ }^{49}$

\footnotetext{
${ }^{1}$ Ames Laboratory and Department of Physics, Iowa State University, Ames IA 50011, USA

${ }^{2}$ Physics Department, Univ. Instelling Antwerpen, Universiteitsplein 1, B-2610 Wilrijk, Belgium and IIHE, ULB-VUB, Pleinlaan 2, B-1050 Brussels, Belgium

and Faculté des Sciences, Univ. de l'Etat Mons, Av. Maistriau 19, B-7000 Mons, Belgium

${ }^{3}$ Physics Laboratory, University of Athens, Solonos Str. 104, GR-10680 Athens, Greece

${ }^{4}$ Department of Physics, University of Bergen, Allégaten 55, N-5007 Bergen, Norway

${ }^{5}$ Dipartimento di Fisica, Università di Bologna and INFN, Via Irnerio 46, I-40126 Bologna, Italy

${ }^{6}$ Collège de France, Lab. de Physique Corpusculaire, IN2P3-CNRS, F-75231 Paris Cedex 05, France

${ }^{7}$ CERN, CH-1211 Geneva 23, Switzerland

${ }^{8}$ Centre de Recherche Nucléaire, IN2P3 - CNRS/ULP - BP20, F-67037 Strasbourg Cedex, France

${ }^{9}$ Institute of Nuclear Physics, N.C.S.R. Demokritos, P.O. Box 60228, GR-15310 Athens, Greece

${ }^{10}$ FZU, Inst. of Physics of the C.A.S. High Energy Physics Division, Na Slovance 2, CS-180 40, Praha 8, Czechoslovakia

${ }^{11}$ Dipartimento di Fisica, Università di Genova and INFN, Via Dodecaneso 33, I-16146 Genova, Italy

${ }^{12}$ Institut des Sciences Nucléaires, IN2P3-CNRS, Université de Grenoble 1, F-38026 Grenoble, France

${ }^{13}$ Research Institute for High Energy Physics, SEFT, P.O. Box 9, FIN-00014 University of Helsinki, Finland

14 Joint Institute for Nuclear Research, Dubna, Head Post Office, P.O. Box 79, 101000 Moscow, Russian Federation

${ }^{15}$ Institut für Experimentelle Kernphysik, Universität Karlsruhe, Postfach 6980, D-76128 Karlsruhe, Germany

${ }^{16}$ High Energy Physics Laboratory, Institute of Nuclear Physics, Ul. Kawiory 26a, PL-30055 Krakow 30 , Poland

${ }^{17}$ Centro Brasileiro de Pesquisas Físicas, rua Xavier Sigaud 150, RJ-22290 Rio de Janeiro, Brazil

${ }^{18}$ Université de Paris-Sud, Lab. de l'Accélérateur Linéaire, IN2P3-CNRS, Bat 200, F-91405 Orsay, France

${ }^{19}$ School of Physics and Materials, University of Lancaster, GB-Lancaster LA1 4YB, UK

${ }^{20}$ LIP, IST, FCUL - Av. Elias Garcia, 14-1 ${ }^{\circ}$, P-1000 Lisboa Codex, Portugal

${ }^{21}$ Department of Physics, University of Liverpool, P.O. Box 147, GB-Liverpool L69 3BX. UK

${ }^{22}$ LPNHE, IN2P3-CNRS, Universités Paris VI et VII, Tour 33 (RdC), 4 place Jussieu, F-75252 Paris Cedex 05, France

${ }^{23}$ Department of Physics, University of Lund, Sölvegatan 14, S-22363 Lund, Sweden

${ }^{24}$ Université Claude Bernard de Lyon, IPNL, IN2P3-CNRS, F-69622 Villeurbanne Cedex, France

${ }^{25}$ Universidad Complutense, Avda. Complutense s/n, E-28040 Madrid, Spain

${ }^{26}$ Univ. d'Aix - Marseille II - CPP, IN2P3-CNRS, F-13288 Marseille Cedex 09, France

${ }^{27}$ Dipartimento di Fisica, Università di Milano and INFN, Via Celoria 16, I-20133 Milan, Italy

${ }^{28}$ Niels Bohr Institute, Blegdamsvej 17, DK-2100 Copenhagen 0, Denmark

${ }^{29} \mathrm{NC}$, Nuclear Centre of MFF, Charles University, Areal MFF, V Holesovickach 2, CS-180 00, Praha 8, Czechoslovakia

${ }^{30}$ NIKHEF-H, Postbus 41882, NL-1009 DB Amsterdam, The Netherlands

${ }^{31}$ National Technical University, Physics Department, Zografou Campus, GR-15773 Athens, Greece

${ }^{32}$ Physics Department, University of Oslo, Blindern, N-1000 Oslo 3, Norway

${ }^{33}$ Dpto. Fisica, Univ. Oviedo, C/P.Jimenez Casas, S/N-33006 Oviedo, Spain

${ }^{34}$ Department of Physics, University of Oxford, Keble Road, Oxford OX1 3RH, UK

${ }^{35}$ Dipartimento di Fisica, Università di Padova and INFN, Via Marzolo 8, I-35131 Padua, Italy

${ }^{36}$ Depto. de Fisica, Pontificia Univ. Católica, C.P. 38071 RJ-22453 Rio de Janeiro, Brazil

37 Rutherford Appleton Laboratory, Chilton, GB - Didcot OX11 OQX, UK

${ }^{38}$ Dipartimento di Fisica, Università di Roma II and INFN, Tor Vergata, I-00173 Rome, Italy

${ }^{39}$ Centre d'Etude de Saclay, DSM/DAPNIA, F-91191 Gif-sur-Yvette Cedex, France

40 Dipartimento di Fisica-Università di Salerno, I-84100 Salerno, Italy

${ }^{41}$ Istituto Superiore di Sanità, Ist. Naz. di Fisica Nucl. (INFN), Viale Regina Elena 299, I-00161 Rome, Italy

${ }^{42}$ C.E.A.F.M., C.S.I.C. - Univ. Cantabria, Avda. los Castros, S/N-39006 Santander, Spain

${ }^{43}$ Inst. for High Energy Physics, Serpukov P.O. Box 35, Protvino, (Moscow Region), Russian Federation

${ }^{44}$ J. Stefan Institute and Department of Physics, University of Ljubljana, Jamova 39, SI-61000 Ljubljana, Slovenia

${ }^{45}$ Fysikum, Stockholm University, Box 6730, S-113 85 Stockholm, Sweden

${ }^{46}$ Dipartimento di Fisica Sperimentale, Università di Torino and INFN, Via P. Giuria 1, I-10125 Turin, Italy

${ }^{47}$ Dipartimento di Fisica, Università di Trieste and INFN, Via A. Valerio 2, I-34127 Trieste, Italy

and Istituto di Fisica, Università di Udine, I-33100 Udine, Italy

${ }^{48}$ Department of Radiation Sciences, University of Uppsala, P.O. Box 535, S-751 21 Uppsala, Sweden

${ }^{49}$ IFIC, Valencia-CSIC, and D.F.A.M.N., U. de Valencia, Avda. Dr. Moliner 50, E-46100 Burjassot (Valencia), Spain

${ }^{50}$ Institut für Hochenergiephysik, Österr. Akad. d. Wissensch., Nikolsdorfergasse 18, A-1050 Vienna, Austria

${ }^{51}$ Inst. Nuclear Studies and University of Warsaw, Ul. Hoza 69, PL-00681 Warsaw, Poland

${ }^{52}$ Fachbereich Physik, University of Wuppertal, Postfach 100 127, D-5600 Wuppertal 1, Germany
} 


\section{Introduction}

An enhancement in the production of identical bosons with similar momenta produced in high energy collisions is observed in any type of reaction (see reference [1] for a review), and attributed to Bose-Einstein (BE) statistics appropriate to identical boson pairs. To study the enhanced probability for emission of two identical bosons it is useful to define a correlation function $R$ :

$$
R\left(p_{1}, p_{2}\right)=\frac{P\left(p_{1}, p_{2}\right)}{P\left(p_{1}\right) P\left(p_{2}\right)}
$$

where $P\left(p_{1}, p_{2}\right)$ is the two-particle probability density, subject to BE symmetrization, and $P\left(p_{i}\right)$ is the corresponding single particle quantity for a particle with four-momentum $p_{i}$. In practice, $P\left(p_{1}\right) P\left(p_{2}\right)$ is often replaced by a reference 2-particle distribution $P_{o}\left(p_{1}, p_{2}\right)$, which, ideally, resembles $P\left(p_{1}, p_{2}\right)$ in all respects, apart from the lack of BE symmetrization. BE correlations can be used to study the space-time structure of the hadronization source [2].

The effect can be described using the variable $Q$, defined by $Q^{2}=M^{2}-4 m^{2}$, where $M$ is the invariant mass of the two particles, and $m$ is the particle mass. The correlation function is usually parametrized as:

$$
R(Q)=1+\lambda e^{-r^{2} Q^{2}}
$$

where the parameter $r$ gives the size of the source and $\lambda$ measures the strength of the correlation between the identical bosons, being 1 for a completely incoherent ("chaotic") source.

This letter describes a study of $\mathrm{K}_{\mathrm{S}}^{0} \mathrm{~K}_{\mathrm{S}}^{0}$ correlations in hadronic $Z^{0}$ decays. When a $\mathrm{K}_{\mathrm{S}}^{0}$ $\mathrm{K}_{\mathrm{S}}^{0}$ pair is produced, there are two possibilities:

a. The $K_{S}^{0} K_{S}^{0}$ pair comes from a $K^{0} K^{0}$ (or $\bar{K}^{0} \bar{K}^{0}$ ) system. In this case, the $K_{S}^{0} K_{S}^{0}$ pair originates from an identical boson pair, and is thus subject to BE symmetrization. At low $Q$ values, where the $B E$ effect is observed, the fraction of $K_{S}^{0} K_{S}^{0}$ coming from identical boson pairs is about $28 \%$ of the whole data sample. This fraction was evaluated using the JETSET 7.3 [3] Monte Carlo program based on a parton shower model (JETSET PS in the following).

b. The $K_{S}^{0} K_{S}^{0}$ pair comes from a $K^{0} \bar{K}^{0}$ system. In this case, the original system is a boson-antiboson pair, and thus is not subject to BE symmetrization. However, as described in detail in reference [4], an enhancement is expected in the low $Q$ region if one selects the $C=+1$ eigenstate of the charge conjugation operator $C$. The wave function of the $\left|\mathrm{K}^{0} \overline{\mathrm{K}}^{0}\right\rangle$ state is a superposition of $C=+1$ and $C=-1$ eigenstates:

$$
\left|\mathrm{K}^{0} \overline{\mathrm{K}}^{0}\right\rangle_{C= \pm 1}=\frac{1}{\sqrt{2}}\left(\left|\mathrm{~K}^{0}(\vec{p}) \overline{\mathrm{K}}^{0}(-\vec{p})\right\rangle \pm\left|\overline{\mathrm{K}}^{0}(\vec{p}) \mathrm{K}^{0}(-\vec{p})\right\rangle\right),
$$

where $\vec{p}$ is the three momentum of one of the kaons in their centre of mass frame. In the limit of $|\vec{p}|=0(Q=0)$, the probability amplitude for the $C=-1$ state $\left(\mathrm{K}_{\mathrm{S}}^{0}\right.$ $\mathrm{K}_{\mathrm{L}}^{0}$ pair) disappears whereas that for the $C=+1$ state $\left(\mathrm{K}_{\mathrm{S}}^{0} \mathrm{~K}_{\mathrm{S}}^{0}\right.$ or $\mathrm{K}_{\mathrm{L}}^{0} \mathrm{~K}_{\mathrm{L}}^{0}$ pair $)$ is maximal. This should cause an enhancement for $\mathrm{K}_{\mathrm{S}}^{0} \mathrm{~K}_{\mathrm{S}}^{0}$ (and $\mathrm{K}_{\mathrm{L}}^{0} \mathrm{~K}_{\mathrm{L}}^{0}$ ) pairs at low $Q$ values, but this is exactly compensated by the low $Q$ suppression of the $\mathrm{K}_{\mathrm{S}}^{0} \mathrm{~K}_{\mathrm{L}}^{0}$ state. This means that no BE effect would be observed (as expected for a boson-antiboson system) if all the possible final states of $\mathrm{K}^{0} \overline{\mathrm{K}}^{0}$ system were detected. Since this analysis uses only $\mathrm{K}_{\mathrm{S}}^{0} \mathrm{~K}_{\mathrm{S}}^{0}$ pairs, one expects to observe a full BE-like enhancement in the low $Q$ region. 
In reality the selected $\mathrm{K}_{\mathrm{S}}^{0} \mathrm{~K}_{\mathrm{S}}^{0}$ sample will not display a full $\mathrm{BE}$ correlation due to the presence of $\mathrm{K}_{\mathrm{S}}^{0}$ coming from sources which are not $\mathrm{BE}$ correlated. In this analysis we correct for non-prompt kaons and for the decays of the $f_{0}(975)$ scalar meson.

Although there are many measurements of BE correlations between pions in the literature including three studies by LEP experiments [5-7], only OPAL has published results on $\mathrm{K}_{\mathrm{S}}^{0} \mathrm{~K}_{\mathrm{S}}^{0}$ interference in $e^{+} e^{-}$interactions [8].

\section{Experimental Procedure}

This study is based on the sample of hadronic events collected with the DELPHI detector at the centre of mass energy around $\sqrt{s}=91.2 \mathrm{GeV}$ in the 1992 running period of LEP. The DELPHI detector has been described in detail elsewhere [9]. The analysis relies on the information provided by the central tracking detectors: the Micro Vertex Detector (VD), the Inner Detector, the Time Projection Chamber (TPC), and the Outer Detector.

The central tracking system of DELPHI covers the region between $25^{\circ}$ and $155^{\circ}$ in the polar angle $\theta$, with reconstruction efficiency close to unity. The average momentum resolution for the charged particles in hadronic final states is in the range $\Delta p / p \simeq 0.001 p$ to $0.01 p(p$ in $\mathrm{GeV} / c)$, depending on which detectors are included in the track fit.

Charged particles were used in the analysis if they had:

- momentum larger than $0.1 \mathrm{GeV} / c$;

- measured track length in the TPC above $30 \mathrm{~cm}$;

- $\theta$ between $25^{\circ}$ and $155^{\circ}$.

Hadronic events were then selected by requiring that:

- the total energy of the charged particles exceeded $15 \mathrm{GeV}$;

- the total energy of the charged particles in each of the two hemispheres defined with respect to the beam axis exceeded $3 \mathrm{GeV}$;

- there were at least 5 charged particles with momenta above $0.2 \mathrm{GeV} / c$.

In the calculation of the energies, all charged particles have been assumed to have the pion mass. A total of 717,511 events satisfied these criteria. The background due to beam-gas scattering, $\gamma \gamma$ interactions and $\tau^{+} \tau^{-}$events has been estimated to be less than $0.3 \%$ of the sample.

The influence of the detector on the analysis was studied with the simulation program DELSIM [10]. Events were generated using JETSET PS, with parameters tuned as in reference [11]. The particles were followed through the detailed geometry of DELPHI giving simulated digitizations in each detector. These data were processed with the same reconstruction and analysis programs as the real data.

\section{Analysis}

The $K_{S}^{0}$ are detected by their decay in flight into $\pi^{+} \pi^{-}$. Such decays are normally separated in space from the $Z^{0}$ decay point (primary vertex), measured for each fill using the VD data.

Candidates for secondary decays, $V_{0}$, were found by considering all pairs of tracks with opposite charge. The vertex of each pair was determined by minimizing the $\chi^{2}$ obtained from the distance of the vertex to the extrapolated tracks.

The $\mathrm{K}_{\mathrm{S}}^{0}$ decay vertex candidates were required to satisfy the following: 
- The probability from the $\chi^{2}$ of the fit was larger than 0.001 .

- In the $R \phi$ plane (perpendicular to the beam direction), the angle between the vector sum of the charged particle momenta and the line joining the primary to the secondary vertex was less than $\left(10+20 / p_{t}\left(\mathrm{~K}_{\mathrm{S}}^{0}\right)\right) \mathrm{mrad}$, where $p_{t}\left(\mathrm{~K}_{\mathrm{S}}^{0}\right)$ is the transverse momentum of the $\mathrm{K}_{\mathrm{S}}^{0}$ candidate relative to the beam axis, in $\mathrm{GeV} / c$.

- The radial separation $r_{R \phi}$ of the primary and secondary vertex in the $R \phi$ plane was larger than four times the error on $r_{R \phi}$ from the fit if VD hits were included in the track fit, six times the error otherwise.

- When the reconstructed decay point of the $K_{S}^{0}$ was beyond the radius of the VD, there were no signals in the VD associated to the decay tracks.

The $\pi^{+} \pi^{-}$invariant mass spectrum from the accepted $\mathrm{K}_{\mathrm{S}}^{0}$ candidates is shown in Figure 1(a). A clear signal of about $111,000 \mathrm{~K}_{\mathrm{S}}^{0}$ is seen over a background of about $17 \%$ within $\pm 10 \mathrm{MeV} / c^{2}$ from the peak. In an interval of $\pm 10 \mathrm{MeV} / c^{2}$ around the nominal $\mathrm{K}^{0}$ mass, the average detection efficiency for $\mathrm{K}_{\mathrm{S}}^{0}$ to $\pi^{+} \pi^{-}$, weighted by the momentum spectrum predicted by JETSET PS, is about $28 \%$.

The background from $\Lambda$ decays into $p \pi^{-}$was found to be flat in the $\pi^{+} \pi^{-}$mass spectrum. The level of this background is about $2 \%$ under the peak. The contribution from photon conversions was found to be negligible.

In order to evaluate the number of $K_{S}^{0}$ pairs, the following procedure was used. First a search was carried out for events with at least two $K_{S}^{0}$ candidates (common tracks were not allowed) with invariant mass within $\pm 100 \mathrm{MeV} / \mathrm{c}^{2}$ of the $\mathrm{K}_{\mathrm{S}}^{0}$ peak. To evaluate the background in this sample, the absolute values $\Delta m_{1}$ and $\Delta m_{2}$ of the differences between the invariant mass of the $\mathrm{K}_{\mathrm{S}}^{0}$ candidate and the known $\mathrm{K}_{\mathrm{S}}^{0}$ mass was computed. The correlation between $\Delta m_{1}$ and $\Delta m_{2}$ is shown in Figure 1(b).

The "signal region", defined by $\Delta m_{1}, \Delta m_{2}<10 \mathrm{MeV} / c^{2}$, contains 14741 pairs coming from 12408 events. The number $N$ of selected pairs is a sum of four contributions:

- $N_{t t}$, the number of pairs of true $\mathrm{K}_{\mathrm{S}}^{0}$;

- $N_{t f}$, the number of pairs in which the first candidate is a true $\mathrm{K}_{\mathrm{S}}^{0}$, while the second is fake;

- $N_{f t}$, the number of pairs in which the first candidate is a fake $\mathrm{K}_{\mathrm{S}}^{0}$, while the second is true;

- $N_{f f}$, the number of pairs in which neither candidate is a true $\mathrm{K}_{\mathrm{S}}^{0}$;

where in the ideal case $N_{f t}=N_{t f}$.

It was verified by simulation that, in the region of $\Delta m$ used for the extrapolation, the background under the $\Delta m_{i}$ peak is flat for each $\mathrm{K}_{\mathrm{S}}^{0}$ candidate. The three background contributions $N_{t f}, N_{f t}$ and $N_{f f}$ can thus be estimated from the numbers of pairs $N_{1}, N_{2}$ and $N_{3}$, in three "control regions" defined in the $\Delta m_{1}, \Delta m_{2}$ correlation plot:

$$
\begin{aligned}
& N_{1}=k N_{t f}+k N_{f f} \quad \Delta m_{1}<a, \quad b<\Delta m_{2}<100 \mathrm{MeV} / c^{2} ; \\
& N_{2}=k N_{f t}+k N_{f f} \quad b<\Delta m_{1}<100 \mathrm{MeV} / c^{2}, \quad \Delta m_{2}<a ; \\
& N_{3}=k^{2} N_{f f} \quad b<\Delta m_{1}<100 \mathrm{MeV} / c^{2}, \quad b<\Delta m_{2}<100 \mathrm{MeV} / c^{2}
\end{aligned}
$$

where

$$
k=\frac{\text { width of the control region }}{\text { width of the signal region }}=\frac{100-b}{a}
$$

The upper limit $a=10 \mathrm{MeV} / c^{2}$ of the signal region and the lower limit $b=20 \mathrm{MeV} / c^{2}$ of the interval defining the control region (from $b$ to $100 \mathrm{MeV} / c^{2}$ ) were chosen to have 
a stable signal to background ratio and to match the hypothesis of a flat background behaviour for the extrapolation procedure.

The number of true pairs in the signal region can then be estimated as:

$$
\begin{aligned}
N_{t t} & =N-\left(N_{t f}+N_{f t}+N_{f f}\right) \\
& =N-\left(N_{1}+N_{2}\right) / k+N_{3} / k^{2},
\end{aligned}
$$

giving finally after background subtraction $10943 \pm 124$ (stat) $\mathrm{K}_{\mathrm{S}}^{0} \mathrm{~K}_{\mathrm{S}}^{0}$ pairs among which $4956 \pm 82($ stat) have $Q<2 \mathrm{GeV} / c$.

Figure 2 shows the distribution as a function of $Q$ of the pairs of $K_{S}^{0}$ for data and simulated events. Simulated events were generated without BE symmetrization. An excess in the experimental data can be observed at low $Q$.

To give a first estimate of the correlation function, we define the ratio $R_{M E A S}(Q)=\mathcal{N}$ $\times N_{R}(Q) / N_{S}(Q)$, where $N_{R}$ and $N_{S}$ are the number of $\mathrm{K}_{\mathrm{S}}^{0}$ pairs per interval of $Q$ for real and simulated data respectively after background subtraction, and $\mathcal{N}$ is a normalization factor computed as the ratio of $N_{S}$ and $N_{R}$ in the range between $0.5 \mathrm{GeV} / c$ and $2 \mathrm{GeV} / c$.

In the case of $\mathrm{K}_{\mathrm{S}}^{0}$ pairs there is no obvious way to extract a good reference sample from the data itself [8], as it is the case of the charged pions [7], so in this analysis a sample of 754,000 simulated events was used as a reference sample.

The correlation function $R_{M E A S}(Q)$ is plotted in Figure 3 . An enhancement is clearly visible in the region $Q<0.4 \mathrm{GeV} / c$.

The best fit to expression (1) gives:

$$
\begin{aligned}
& \lambda=0.88 \pm 0.35(\text { stat }) \pm 0.16(\text { syst }) \\
& r=0.82 \pm 0.16(\text { stat }) \pm 0.12(\text { syst }) \mathrm{fm},
\end{aligned}
$$

with $\chi^{2}=8.5$ for 10 degrees of freedom and correlation coefficient 0.62 . The evaluation of systematic errors is explained in section 3.2 .

In Figure 4, the contour plot of the $68 \%$ and $90 \%$ confidence levels for the fit of $R_{M E A S}$ to (1) is shown. Results from OPAL [8] on $\mathrm{K}_{\mathrm{S}}^{0} \mathrm{~K}_{\mathrm{S}}^{0}$ correlations and results from LEP [5-7] on two-pion correlations are also shown.

\subsection{Corrections for non-interfering kaons}

Due to the presence of $K_{S}^{0}$ not coming from the primary interaction, the measured correlation between $\mathrm{K}_{\mathrm{S}}^{0}$ pairs is expected to be smaller than the bare correlation between prompt $K_{S}^{0}$.

In particular, a non-negligible fraction of $\mathrm{K}_{\mathrm{S}}^{0}$ is expected to come from the decay of charmed and bottom particles. In order to perform this correction we need to estimate the fraction $f_{b c}(Q)$ of $\mathrm{K}_{\mathrm{S}}^{0} \mathrm{~K}_{\mathrm{S}}^{0}$ pairs including at least one $\mathrm{K}_{\mathrm{S}}^{0}$ coming from the decays of charmed or bottom particles. $f_{b c}(Q)$ was computed by means of JETSET PS, and is displayed in Table $1^{\dagger}$.

Due to the correction $f_{b c}(Q)$, the correlation function becomes

$$
R^{*}(Q)=1+\frac{R_{M E A S}(Q)-1}{\left(1-f_{b c}(Q)\right)} .
$$

\footnotetext{
${ }^{\dagger}$ The rise of $f_{b c}(Q)$ at low $Q$ values is due to pairs from the same decay. For most of these pairs the decay amplitude is highly constrained and thus they should not display BE enhancement (coherent source) [12]. Under the extreme assumption that all pairs from a common ancestor would fully interfere, $\lambda$ would be decreased by $20 \%$. This is anyway an overestimate of the effect.
} 
A fit of $R^{*}$ to equation 1 yielded:

$$
\begin{aligned}
& \lambda^{*}=1.31 \pm 0.53(\text { stat }) \pm 0.22(\text { syst }) \\
& r^{*}=0.84 \pm 0.16(\text { stat }) \pm 0.10(\text { syst }) \mathrm{fm} .
\end{aligned}
$$

A correction to $R^{*}(Q)$ is needed due to the absence of decays of mesons like $f_{0}(975)$ and $a_{0}(980)$ in the simulated sample. Such decays can fake the correlation effect, producing two $K_{S}^{0}$ very close in momentum. The production cross section at LEP energies is presently known only for $f_{0}$ [13]. The mean multiplicity of $f_{0}$ per hadronic event has been measured to be $0.10 \pm 0.04$ in the region of momentum of the $f_{0}$ larger than 4.5 $\mathrm{GeV} / \mathrm{c}$. We could thus correct only for this source.

The $Q$ distribution for $\mathrm{K} \overline{\mathrm{K}}$ pairs coming from $f_{0}$ decay was calculated by using the coupled channel Breit-Wigner formula [14] including the $\mathrm{K} \overline{\mathrm{K}}$ threshold effects. The $f_{0}$ branching ratio to kaons was taken from reference [15]. The correlation function $R^{* *}(Q)$ after subtraction of these pairs is listed in Table 1.

A fit of $R^{* *}$ to equation 1 yielded ${ }^{\ddagger}$ :

$$
\begin{aligned}
& \lambda^{* *}=1.13 \pm 0.54(\text { stat }) \pm 0.23(\text { syst }) \\
& r^{* *}=0.90 \pm 0.19(\text { stat }) \pm 0.10(\text { syst }) \mathrm{fm} .
\end{aligned}
$$

\begin{tabular}{|l|c|c|c|c|}
\hline$Q(\mathrm{GeV} / \mathrm{c})$ & $R_{M E A S}(Q)$ & $f_{b c}(Q)$ & $R^{*}(Q)$ & $R^{* *}(Q)$ \\
\hline $0.000-0.167$ & $1.69 \pm 0.34$ & 0.328 & $2.03 \pm 0.51$ & $1.86 \pm 0.50$ \\
$0.167-0.333$ & $1.34 \pm 0.12$ & 0.287 & $1.48 \pm 0.17$ & $1.37 \pm 0.18$ \\
$0.333-0.500$ & $1.01 \pm 0.07$ & 0.243 & $1.02 \pm 0.09$ & $0.97 \pm 0.10$ \\
$0.500-0.667$ & $0.99 \pm 0.06$ & 0.238 & $0.98 \pm 0.08$ & $0.96 \pm 0.08$ \\
$0.667-0.833$ & $1.05 \pm 0.06$ & 0.232 & $1.07 \pm 0.08$ & $1.06 \pm 0.08$ \\
$0.833-1.000$ & $0.93 \pm 0.06$ & 0.223 & $0.91 \pm 0.07$ & $0.91 \pm 0.07$ \\
$1.000-1.167$ & $1.02 \pm 0.06$ & 0.211 & $1.03 \pm 0.08$ & $1.03 \pm 0.08$ \\
$1.167-1.333$ & $0.91 \pm 0.06$ & 0.235 & $0.89 \pm 0.08$ & $0.89 \pm 0.08$ \\
$1.333-1.500$ & $1.14 \pm 0.08$ & 0.246 & $1.19 \pm 0.10$ & $1.20 \pm 0.10$ \\
$1.500-1.667$ & $0.98 \pm 0.08$ & 0.236 & $0.97 \pm 0.10$ & $0.98 \pm 0.10$ \\
$1.667-1.833$ & $0.99 \pm 0.08$ & 0.231 & $0.98 \pm 0.10$ & $1.00 \pm 0.10$ \\
$1.833-2.000$ & $1.01 \pm 0.08$ & 0.246 & $1.01 \pm 0.11$ & $1.02 \pm 0.11$ \\
\hline
\end{tabular}

Table 1: Correlation function before and after corrections. $R_{M E A S}(Q)$ is the measured value; $R^{*}(Q)$ is the value after correcting for the contribution of non-prompt kaons $f_{b c}(Q)$; and in $R^{* *}(Q)$ the $f_{0}$ correction is also applied.

\subsection{Evaluation of the Systematic Error}

The following sources of systematic error were considered.

- Reference sample

In order to estimate the systematics of the reference sample, we varied the physical parameters of the simulation. Relevant parameters for the parton shower evolution of JETSET PS are the QCD parameter $\Lambda$, and the cut-off parameter $Q_{0}$. Their

${ }^{\ddagger}$ A rough estimate of the $a_{0}(980)$ contribution (assuming that the $a_{0}$ multiplicity is the average of the predictions from JETSET PS and HERWIG, and that the $a_{0}$ behaves like the $f_{0}$ ) would decrease $\lambda^{* *}$ by approximately $10 \%$. 
ranges of variations were established as in reference [16]: $\Lambda$ was allowed to vary between 0.25 and $0.32 \mathrm{GeV}$, and $Q_{0}$ in the range between 0.6 and $1.4 \mathrm{GeV}$.

The fragmentation in JETSET PS is governed by the Lund symmetric fragmentation function, with essentially only one free parameter, a. Its value was found in reference [16] to be between 0.14 and 0.26 (the $b$ parameter was fixed at $0.34 \mathrm{GeV}^{-2}$ ). The transverse momentum of primary hadrons is parametrized by a Gaussian, whose width $\sigma_{q}$ was varied between $355 \mathrm{MeV} / c$ and $415 \mathrm{MeV} / c$.

In addition, the following were varied:

- the probability of generating a prompt spin 1 meson, $V /(V+P)$, between 0.47 and 0.67 . This range was calculated from the $K^{0}$ and $K^{*}$ average multiplicities measured previously by DELPHI $[17,13]$.

- the probability of exciting a $s \bar{s}$ pair from the vacuum, $\gamma_{s} / \gamma_{u}$, between 0.28 and 0.32 . This range was derived [17] from the $\mathrm{K}^{0}$ differential cross section.

The uncertainties due to these sources are shown in Table 2.

\begin{tabular}{|l|c|c|c|c|c|c|}
\hline & \multicolumn{2}{|c|}{ Uncorr. data } & \multicolumn{2}{|c|}{ After $f_{b c}$ corr. } & \multicolumn{2}{c|}{ After $f_{b c}$ and $f_{0}$ corr. } \\
\hline Source & $\Delta \lambda$ & $\Delta r(\mathrm{fm})$ & $\Delta \lambda^{*}$ & $\Delta r^{*}(\mathrm{fm})$ & $\Delta \lambda^{* *}$ & $\Delta r^{* *}(\mathrm{fm})$ \\
\hline$\sigma_{q}$ & \pm 0.073 & \pm 0.084 & \pm 0.092 & \pm 0.063 & \pm 0.107 & \pm 0.066 \\
$\mathrm{a}$ & \pm 0.037 & \pm 0.020 & \pm 0.046 & \pm 0.016 & \pm 0.053 & \pm 0.020 \\
$\Lambda$ & \pm 0.021 & \pm 0.005 & \pm 0.026 & \pm 0.003 & \pm 0.025 & \pm 0.005 \\
$Q_{0}$ & \pm 0.048 & \pm 0.029 & \pm 0.059 & \pm 0.023 & \pm 0.053 & \pm 0.022 \\
$\gamma_{s} / \gamma_{u}$ & \pm 0.014 & \pm 0.014 & \pm 0.017 & \pm 0.011 & \pm 0.019 & \pm 0.009 \\
$\mathrm{~V} /(\mathrm{V}+\mathrm{P})$ & \pm 0.039 & \pm 0.066 & \pm 0.048 & \pm 0.050 & \pm 0.044 & \pm 0.049 \\
\hline Total & \pm 0.11 & \pm 0.11 & \pm 0.13 & \pm 0.09 & \pm 0.14 & \pm 0.09 \\
\hline
\end{tabular}

Table 2: Systematic errors from different sources contributing to the uncertainty due to the reference sample. Contributions to the corrected correlation functions are shown in the four last columns.

- Selection of the pairs

The systematics were evaluated by a variation of the signal region upper limit from $a=8 \mathrm{MeV} / c^{2}$ to $20 \mathrm{MeV} / c^{2}$, and of the control region from the range between 10 and $100 \mathrm{MeV} / \mathrm{c}^{2}$ off the mass peak, to the range between 40 and $100 \mathrm{MeV} / \mathrm{c}^{2}$ off the mass peak. During the variation the upper limit of the signal region was required to be less than the lower limit of the control region.

- Normalization of $\mathrm{R}$

This source was estimated by varying the range of $Q$ used for the normalization; the intervals of $Q$ between 0.333 and $2 \mathrm{GeV} / c$, and between 1 and $2 \mathrm{GeV} / c$ were studied.

- Non-prompt kaons

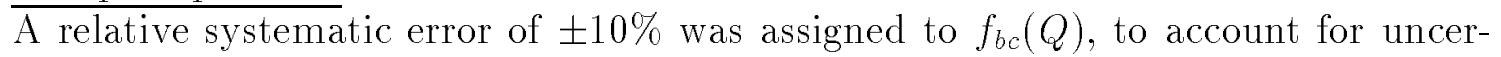
tainties in the decay modes of charmed and bottom particles.

- $f_{0}$ production

The multiplicity of $f_{0}$-mesons per hadronic event was varied within errors of the DELPHI measurement $(0.10 \pm 0.04)$.

The total systematic errors were calculated by adding in quadrature all the contributions. 


\begin{tabular}{|l|c|c|c|c|c|c|}
\hline & \multicolumn{2}{|c|}{ Uncorr. data } & \multicolumn{2}{|c|}{ After $f_{b c}$ corr. } & After $f_{b c}$ and $f_{0}$ corr. \\
\hline Source & $\Delta \lambda$ & $\Delta r(\mathrm{fm})$ & $\Delta \lambda^{*}$ & $\Delta r^{*}(\mathrm{fm})$ & $\Delta \lambda^{* *}$ & $\Delta r^{* *}(\mathrm{fm})$ \\
\hline Reference sample (See Table 2$)$ & \pm 0.11 & \pm 0.11 & \pm 0.13 & \pm 0.09 & \pm 0.14 & \pm 0.09 \\
Selection of the pairs & \pm 0.11 & \pm 0.04 & \pm 0.17 & \pm 0.04 & \pm 0.16 & \pm 0.04 \\
Normalization of $\mathrm{R}$ & \pm 0.01 & \pm 0.01 & \pm 0.05 & \pm 0.01 & \pm 0.01 & \pm 0.01 \\
$10 \%$ relative error of $f_{b c}(Q)$ & - & - & \pm 0.06 & \pm 0.00 & \pm 0.06 & \pm 0.00 \\
$\left\langle n_{f_{0}(975)}\right\rangle=0.10 \pm 0.04$ & - & - & - & - & \pm 0.07 & \pm 0.03 \\
\hline Total systematic error & \pm 0.16 & \pm 0.12 & \pm 0.22 & \pm 0.10 & \pm 0.23 & \pm 0.10 \\
\hline
\end{tabular}

Table 3: Systematic errors.

\section{Conclusions}

Low- $Q$ correlations between pairs of $\mathrm{K}_{\mathrm{S}}^{0}$ from the decay of the $Z^{0}$ have been observed with the DELPHI detector at LEP, using a sample of about 11,000 pairs coming from 717.511 selected hadronic events. The results show a BE-like enhancement which can be parametrized by the expression $R(Q)=1+\lambda e^{-r^{2} Q^{2}}$, where, without any correction for non-prompt $\mathrm{K}_{\mathrm{S}}^{0}$ pairs, $\lambda=0.88 \pm 0.35($ stat $) \pm 0.16($ syst $)$ and $r=0.82 \pm 0.16($ stat $) \pm$ 0.12 (syst) fm.

Taking into account the contribution from non-prompt $K_{S}^{0}$ pairs coming from the decays of charmed and bottom hadrons changes the values above to $\lambda=1.31 \pm 0.53($ stat $) \pm$ $0.22($ syst $)$ and $r=0.84 \pm 0.16($ stat $) \pm 0.10($ syst $) \mathrm{fm}$. Taking into account in addition the decay of the $f_{0}$ into $\mathrm{K} \overline{\mathrm{K}}$ pairs gives $\lambda=1.13 \pm 0.54($ stat $) \pm 0.23$ (syst) and $r=$ $0.90 \pm 0.19($ stat $) \pm 0.10($ syst $) \mathrm{fm}$.

The radius of the region of emission of the kaons compares with that measured by DELPHI for the pions [7], $r=0.62 \pm 0.04$ (stat) \pm 0.20 (syst) fm. The physical interpretation of a comparison of the strength of the correlations is more difficult, since the results in reference [7] were not corrected for particles coming from long-living secondary sources (like B and D mesons). A new analysis by DELPHI [18] makes a correction for all these sources, obtaining a value of $\lambda$ consistent with 1 .

According to JETSET PS, $28 \%$ of the low $Q \mathrm{~K}_{\mathrm{S}}^{0} \mathrm{~K}_{\mathrm{S}}^{0}$ pairs come from identical $\mathrm{K}^{0} \mathrm{~K}^{0}$ (or $\overline{\mathrm{K}}^{0} \overline{\mathrm{K}}^{0}$ ) pairs; about $70 \%$ of them originate from prompt sources. If the positive interference at low $Q$ would affect only the $\mathrm{K}^{0} \mathrm{~K}^{0}$ (or $\overline{\mathrm{K}}^{0} \overline{\mathrm{K}}^{0}$ ) pairs, a value of $\lambda \simeq 3-5$ would be required to explain our data. Assuming that the strength of the Bose-Einstein effect can be parametrized by $\lambda \leq 1$, it is then unlikely that only identical neutral kaons are responsible for the observed enhancement at low $Q$. Our results support the hypothesis that $\mathrm{K}_{\mathrm{S}}^{0}$ coming from $\mathrm{K}^{0} \overline{\mathrm{K}}^{0}$ pairs also exhibit constructive interference at $Q \simeq 0$.

\section{Acknowledgments}

We are greatly indebted to our technical staffs and collaborators and funding agencies for their support in building the DELPHI detector and to the many members of the CERN-SL division for the excellent performance of the LEP machine. We would like to thank M.G. Bowler and N. Törnqvist for useful discussions. 


\section{References}

[1] B. Lörstad, Int. J. Mod. Phys. A4 (1989) 2861.

W. A. Zajc, in "Hadronic Multiparticle Production", P. Carruthers ed., World Scientific (1988) 235.

B. De Lotto, Contributed talk of the XXVI International Conference in High Energy Physics, Dallas, August 1992.

[2] G.I. Kopylov and M.I. Podgoretskii, Sov. J. Nucl. Phys. 19 (1974) 215; G.I. Kopylov, Phys. Lett. 50B (1974) 472.

G. Cocconi, Phys. Lett. 49B (1974) 459.

M. Deutschmann et al., Nucl. Phys. 204B (1982) 333.

A. Giovannini and G. Veneziano, Nucl Phys. B130 (1977) 61.

M. G. Bowler, Z. Phys. C29 (1985) 617.

[3] T. Sjöstrand, Comp. Phys. Comm. 27 (1982) 243, ibid. 28 (1983) 229.

T. Sjöstrand and M. Bengtsson, Comp. Phys. Comm. 43 (1987) 367.

T. Sjöstrand, CERN-TH.6488/92, May 1992.

[4] H. Lipkin, Phys. Lett. B219 (1989) 474; H. Lipkin, Phys. Rev. Lett. 69 (1992) 3700.

[5] P.D. Acton et al. (OPAL Collaboration), Phys. Lett. 267B (1991) 143.

[6] D. Decamp et al. (ALEPH Collaboration), Z. Phys. C54 (1992) 75.

[7] P. Abreu et al. (DELPHI Collaboration), Phys. Lett. B286 (1992) 201.

[8] P. D. Acton et al. (OPAL Collaboration), Phys. Lett. 298B (1993) 456.

[9] P. Aarnio et al. (DELPHI Collaboration), Nucl. Instr. and Meth. A303 (1991) 233.

[10] DELSIM User Manual, DELPHI 87-96 PROG-99, Geneva, July 1989. DELSIM Reference Manual, DELPHI 87-98 PROG-100, Geneva, July 1989.

[11] W. de Boer and H. Furstenau, IEKP-KA/91-07, Karlsruhe, June 1991.

[12] M. G. Bowler, Z. Phys. C46 (1990) 305.

[13] P. Abreu et al. (DELPHI Collaboration), Phys. Lett. 298B (1993) 236.

[14] T.A. Armstrong et al. (WA 76 Collaboration), Z. Phys. C51 (1991) 351.

[15] M. Aguilar-Benitez et al. (Particle Data Group), Phys. Rev. D45 (1992).

[16] P. Abreu et al. (DELPHI Collaboration), Phys. Lett. 295B (1992) 383.

[17] P. Abreu et al. (DELPHI Collaboration), Phys. Lett. 275B (1992) 231.

[18] P. Abreu et al. (DELPHI Collaboration) "Invariant Mass Dependence of Particle Correlations in Hadronic Final States from the Decay of the Z", to be published. 

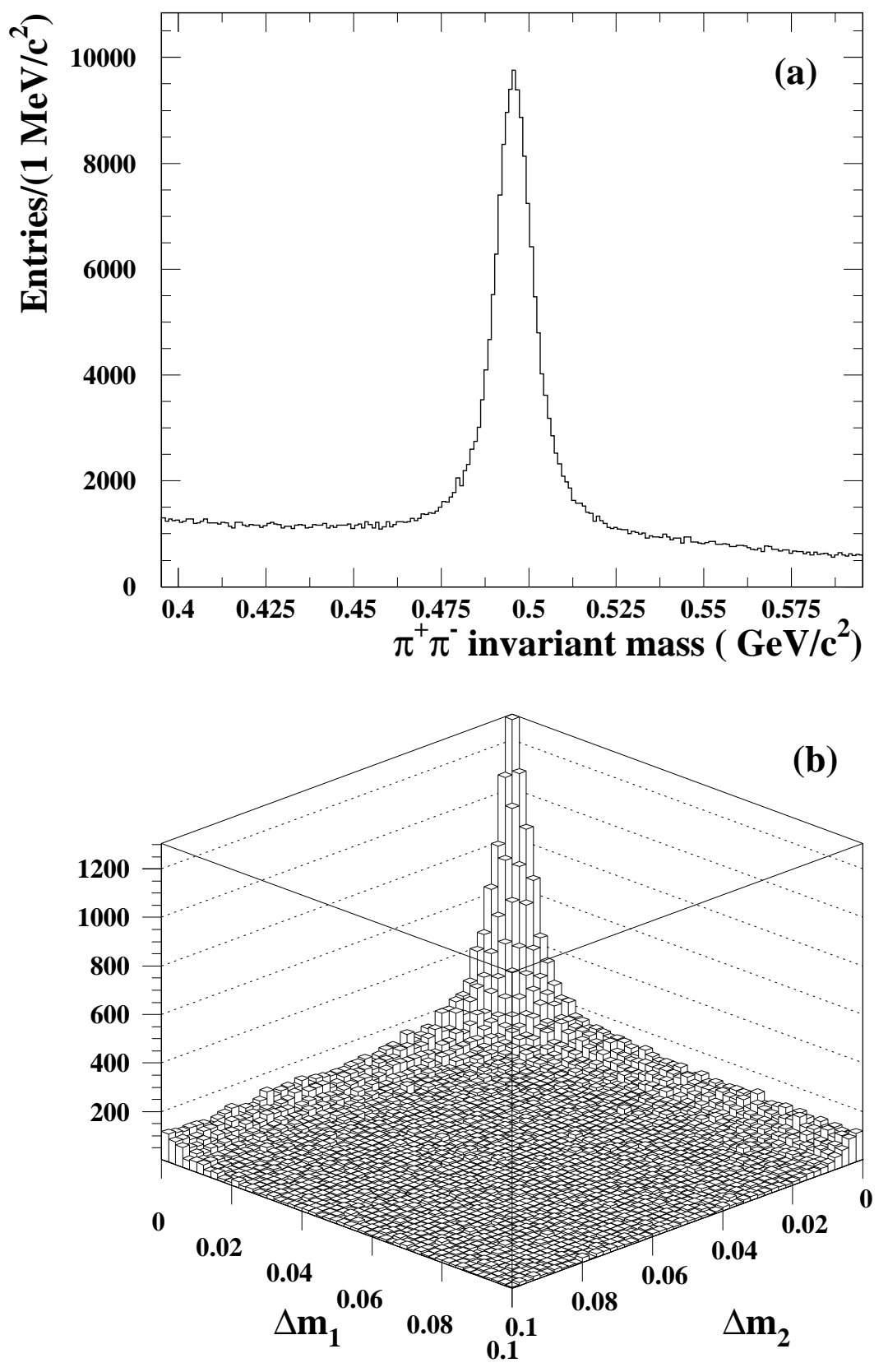

Figure 1: (a) $\pi^{+} \pi^{-}$invariant mass spectrum for the accepted secondary vertices used in the present analysis. (b) Two-dimensional plot of the absolute value of the difference from the nominal $\mathrm{K}_{\mathrm{S}}^{0}$ mass $\Delta \mathrm{m}\left(\mathrm{GeV} / c^{2}\right)$ when two or more $\mathrm{K}_{\mathrm{S}}^{0}$ candidates are present in the same event. 


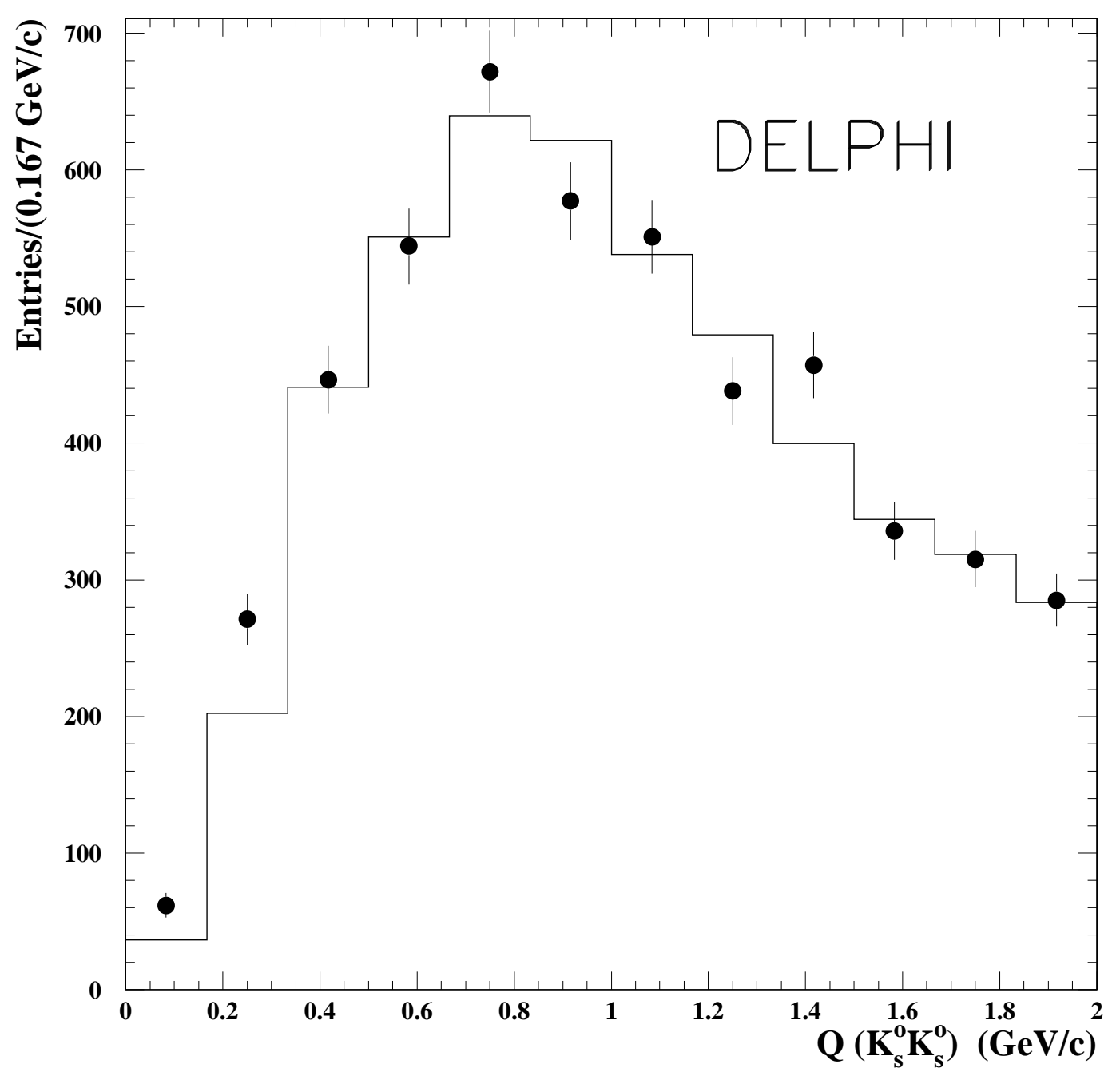

Figure 2: Distribution of $K_{S}^{0}$ pairs in data (points) and simulation without Bose-Einstein interference (solid line), as a function of $Q$. The contents of the simulated sample is normalized to be equal to the data sample in the range $0.5-2.0 \mathrm{GeV} / c^{2}$. 


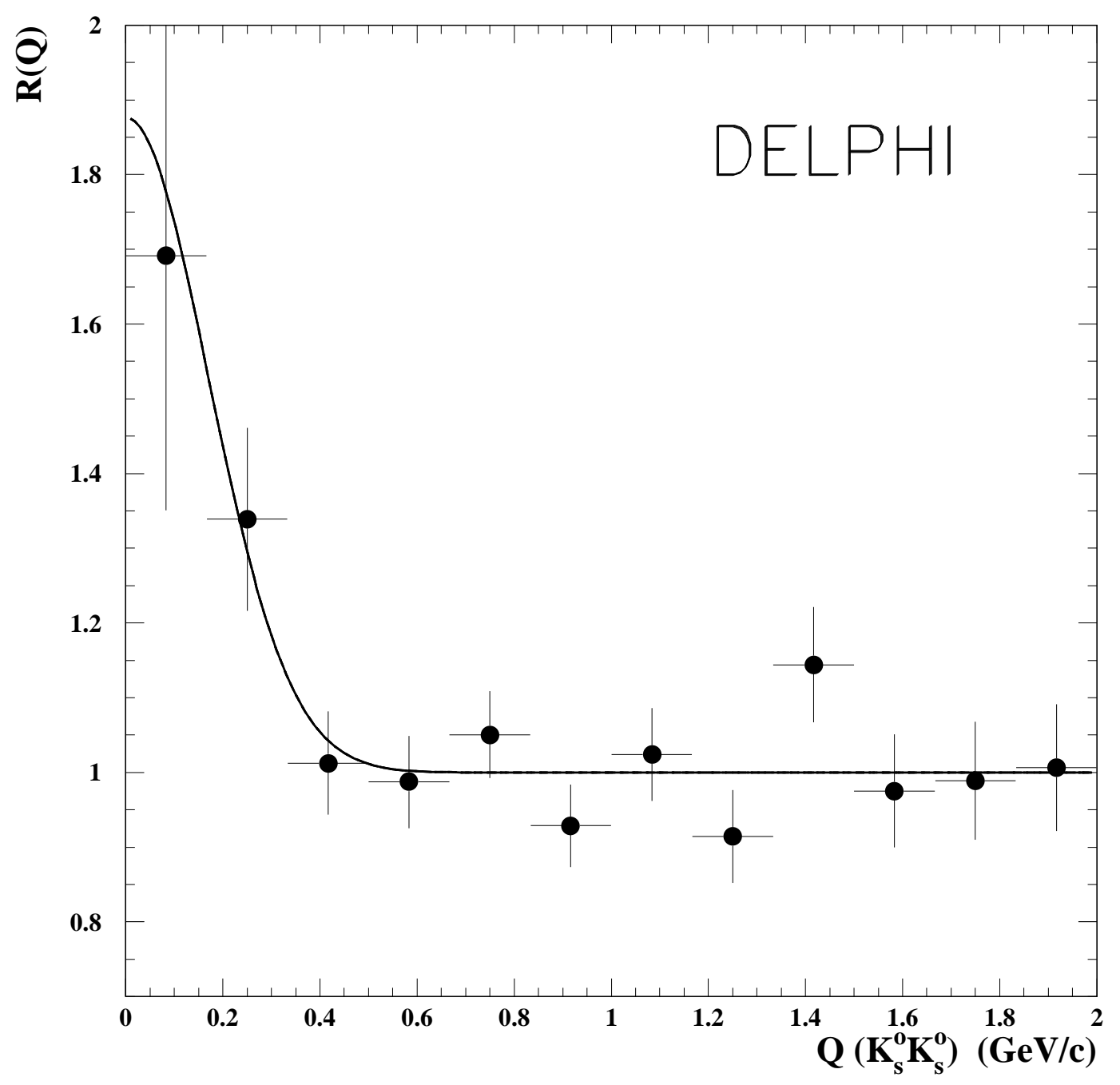

Figure 3: Ratio $R_{M E A S}(Q)$. The best fit to (1) is superimposed as a solid line. 


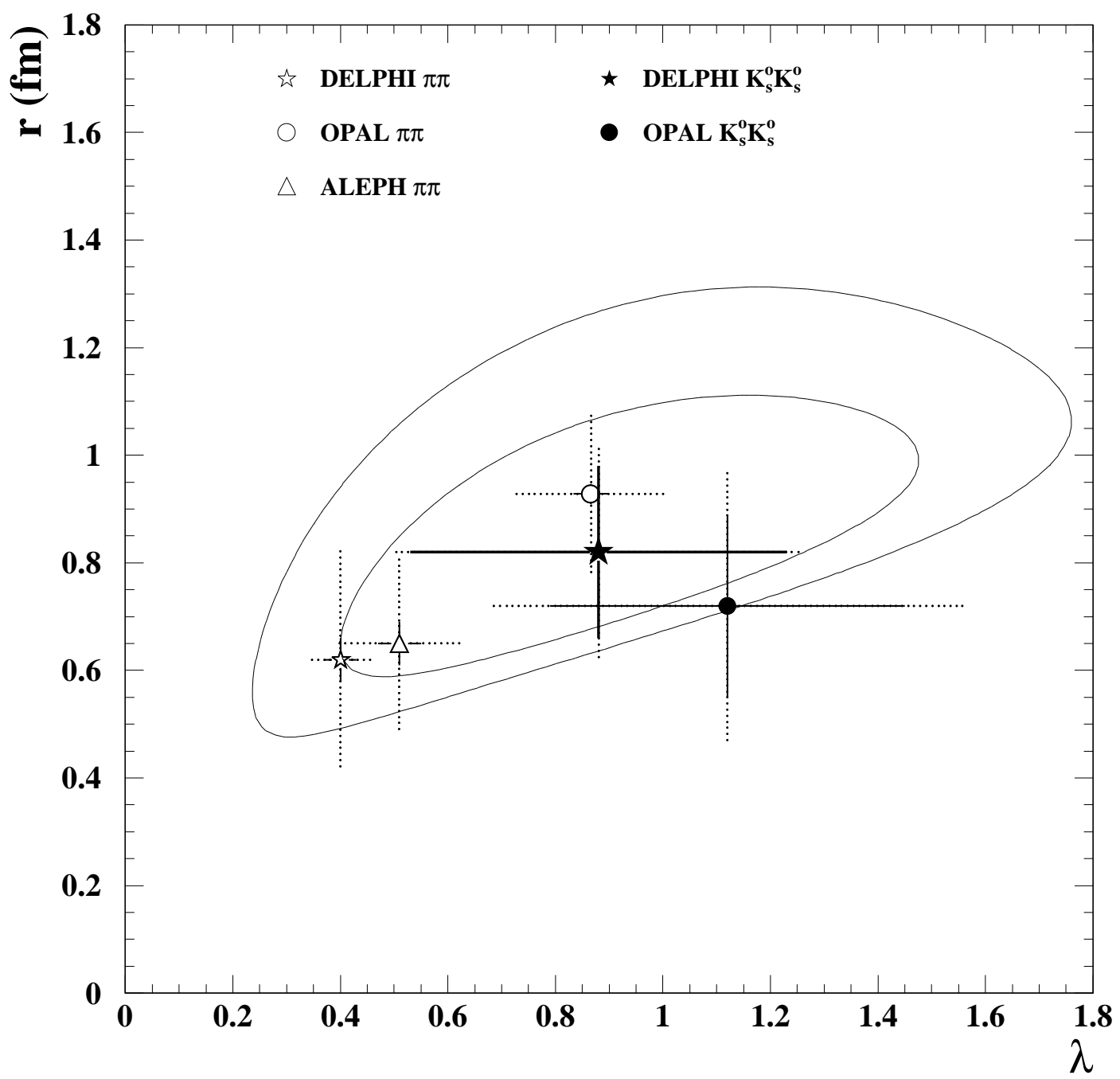

Figure 4: The inner line shows the $68 \%$ and the outer line shows the $90 \%$ confidence level for the fit of $R_{M E A S}$ to (1); statistical error only. OPAL results [8] on $R_{M E A S}$ from $\mathrm{K}_{\mathrm{S}}^{0} \mathrm{~K}_{\mathrm{S}}^{0}$ correlations are also shown. Note that for $R_{M E A S}$, corrections for non-prompt $\mathrm{K}_{\mathrm{S}}^{0}$ and for $f_{0}(975)$ and $a_{0}(980)$ production have not been applied. Results from LEP [5-7] on two-pion correlations, displayed in the same plot, have different correction procedures for non-interfering pairs, and different reference samples. The extra dotted error bars correspond to the systematic errors added in quadrature. 